\title{
Criminologie
}

\section{La situation des mères incarcérées et de leurs enfants au Québec}

\section{Brigitte Blanchard}

Volume 35, numéro 2, automne 2002

Femmes et enfermement au Canada : une décennie de réformes

URI : https://id.erudit.org/iderudit/008292ar

DOI : https://doi.org/10.7202/008292ar

Aller au sommaire du numéro

\section{Éditeur(s)}

Les Presses de l'Université de Montréal

ISSN

0316-0041 (imprimé)

1492-1367 (numérique)

Découvrir la revue

Citer cet article

Blanchard, B. (2002). La situation des mères incarcérées et de leurs enfants au Québec. Criminologie, 35(2), 91-112. https://doi.org/10.7202/008292ar

\section{Résumé de l'article}

Jusqu'ici, peu d'études québécoises ont traité de la question, fort complexe, des relations mère-enfant en milieu carcéral. Dans la mesure où de nouveaux programmes visant à favoriser ces liens ont été mis en place au cours des dernières années, cette question mérite pourtant d'être approfondie. Fruit d'une recherche effectuée dans le cadre d'un mémoire de maîtrise, cet article vise à présenter un portrait de la situation des mères incarcérées et de leurs enfants et à examiner les relations entretenues au cours de la sentence carcérale. Les données présentées ici ont été recueillies à l'aide d'un questionnaire distribué dans les principaux établissements carcéraux et maisons de transition pour femmes du Québec. À ces données se sont greffés différents éléments d'analyse obtenus par observation ou lors d'entretiens avec les différents acteurs impliqués auprès de ces femmes. À l'analyse, on constate que les détenues ayant des enfants présentent plusieurs caractéristiques semblables à celles qui se dégagent pour l'ensemble des femmes incarcérées (faibles revenus, sous-scolarisation, problèmes de toxicomanie, etc.). On remarque ensuite que les services et programmes pour maintenir le lien mère-enfant varient considérablement d'un établissement à l'autre et semblent répondre aux besoins d'une minorité. De tels constats mettent en lumière la complexité de la problématique et la nécessité de poursuivre les recherches afin de cibler les priorités d'action permettant d'assurer le meilleur intérêt de la dyade mère-enfant.
Ce document est protégé par la loi sur le droit d'auteur. L'utilisation des services d'Érudit (y compris la reproduction) est assujettie à sa politique d'utilisation que vous pouvez consulter en ligne.

https://apropos.erudit.org/fr/usagers/politique-dutilisation/ 


\title{
La situation des mères incarcérées et de leurs enfants au Québec
}

\author{
Brigitte Blanchard ${ }^{1}$ \\ Candidate à la maîtrise \\ École de criminologie \\ Université de Montréal \\ brigitte.blanchard@sympatico.ca
}

RÉSUMÉ • Jusqu'ici, peu d'études québécoises ont traité de la question, fort complexe, des relations mère-enfant en milieu carcéral. Dans la mesure où de nouveaux programmes visant à favoriser ces liens ont été mis en place au cours des dernières années, cette question mérite pourtant d'être approfondie. Fruit d'une recherche effectuée dans le cadre d'un mémoire de maîtrise, cet article vise à présenter un portrait de la situation des mères incarcérées et de leurs enfants et à examiner les relations entretenues au cours de la sentence carcérale. Les données présentées ici ont été recueillies à l'aide d'un questionnaire distribué dans les principaux établissements carcéraux et maisons de transition pour femmes du Québec. À ces données se sont greffés différents éléments d'analyse obtenus par observation ou lors d'entretiens avec les différents acteurs impliqués auprès de ces femmes. À l'analyse, on constate que les détenues ayant des enfants présentent plusieurs caractéristiques semblables à celles qui se dégagent pour l'ensemble des femmes incarcérées (faibles revenus, sous-scolarisation, problèmes de toxicomanie, etc.). On remarque ensuite que les services et programmes pour maintenir le lien mère-enfant varient considérablement d'un établissement à l'autre et semblent répondre aux besoins d'une minorité. De tels constats mettent en lumière la complexité de la problématique et la nécessité de poursuivre les recherches afin de cibler les priorités d'action permettant d'assurer le meilleur intérêt de la dyade mère-enfant.

ABSTRACT - Until now, few studies in Quebec have dealt with the complex issue of motherchild relationship in jail. As many new programs aimed at strengthening this relation-

1. Cet article présente une partie des résultats d'une recherche réalisée dans le cadre d'un mémoire de maîtrise. L'auteure est employée du Service correctionnel du Canada. Nous tenons toutefois à préciser que les opinions et conclusions présentées ici ne sont pas nécessairement celles du ministère du Solliciteur général du Canada. Nous voudrions remercier M. Guy Lemire et $\mathrm{M}$. Carlo Morselli, de l'École de criminologie, pour leur précieuse aide à la réalisation de cette recherche. Un merci tout spécial à Denis Béliveau pour ses commentaires et à Claude Legendre, traductrice. 
ship were put in place over the last few years, the issue would be worth some consideration. A synthesis of a research done for a master's thesis, the purpose of this paper is to draw a profile of incarcerated mothers and their children in Quebec and to study their relationship during confinement. To collect these data, we distributed a questionnaire among Quebec's main women's prisons and half-way houses. We also included data gathered during our on-site visits and interviews with the various parties involved. After analysis, the study indicated that these mothers show many similarities (low income, low education level, drug addiction...) with the overall population of incarcerated women. It becomes evident that the programs and services aimed at maintaining the mother-child relationship are very different between penal institutions and seem to address the needs of only a minority. These findings put emphasis on the problematic and the need to carry on studies on the whole issue if we are to determine priorities of interventions that truly serve the best interest of the mother-child dyad.

\section{Introduction}

La présence d'enfants en milieu carcéral constitue une pratique correctionnelle novatrice visant à permettre aux détenues qui sont mères de maintenir ou de favoriser leurs contacts avec leur(s) enfant(s). Au Québec, une telle pratique est devenue réalité en 1998 à l'établissement fédéral pour femmes de Joliette. Dans cet article, nous voudrions brosser un tableau de la situation des mères incarcérées et de leur(s) enfant(s) au Québec et nous pencher sur la façon dont ces dernières actualisent les contacts avec ceux-ci au cours de leur sentence carcérale. Par la même occasion, nous examinerons comment les structures institutionnelles répondent à leurs besoins afin de pouvoir suggérer un certain nombre de pistes d'intervention.

\section{L'état de la question}

Au Canada, l'intégration des enfants au sein de l'univers carcéral constitue une pratique encore peu répandue. Entre 1934 et le milieu des années 1990, le seul pénitencier ${ }^{2}$ pour femmes au Canada était situé à Kingston, en Ontario. Dans cet établissement, les contacts que pouvaient avoir les mères avec leurs enfants se limitaient aux visites régulières et aux visites familiales privées; programmes qui, en fait, étaient accordés à tous les délinquants séjournant dans les pénitenciers canadiens. Les mères détenues

2. Au Canada, les pénitenciers abritent les personnes condamnées à des peines de 2 ans et plus. Ces établissements relèvent de l'administration fédérale. Les prisons accueillent les individus condamnés à des peines de moins de 2 ans et relèvent de l'administration des provinces. 
pouvaient donc recevoir la visite de leurs enfants, toujours accompagnés d'un adulte autorisé, au parloir et/ou derrière une vitre (visite sécuritaire) ou encore les recevoir à une fréquence bimestrielle dans des petites unités isolées pour des séjours de trois jours (Bertrand et al., 1998; Fournier 2000 ).

La situation pour les femmes purgeant une peine fédérale a toutefois commencé à évoluer, vers le milieu des années 1990, alors que le service correctionnel du Canada ont mis en place cinq établissements régionaux munis de petites unités de logement de type unifamilial dispersées sur de vastes terrains (SCC, 1990 : 103). Cette nouvelle approche correctionnelle vise en fait à recréer un hébergement se rapprochant d'un idéal fondé sur la communauté afin de permettre aux femmes de faire des choix responsables et valables quant à leur réinsertion sociale et «les soutenir dans leurs responsabilités envers leurs enfants et les autres membres de la famille» (SCC, 1990 : 144). C'est donc dans ce contexte fort différent des prisons traditionnelles que la cohabitation mère-enfant est maintenant possible. Quant aux prisons canadiennes, hormis quelques programmes de visites mère-enfant et deux prisons de l'Ouest canadien permettant la cohabitation des enfants jusqu'à l'âge, respectivement, de 10 mois et de 3 ans (MacLeod, 1986; MacLean, 1997 ; Bertrand et al., 1998), on y retrouve très peu de services adaptés à leurs besoins.

Lorsqu' on examine ce qui se fait à l'extérieur du Canada, on constate qu'en Europe et aux États-Unis, une panoplie de programmes et de services se sont développés depuis plusieurs décennies afin de favoriser et/ou restaurer les relations mères-enfants. Les programmes de cohabitation en milieu carcéral se retrouvent dans plusieurs prisons européennes pour femmes mais l'accès à ces programmes n'est pas partout le même. Ils s'actualisent, dans plusieurs régions, en marge de la population générale, dans des lieux spécifiquement aménagés pour les enfants, et ne s'adressent qu'aux enfants d'âge préscolaire pour des séjours à durée limitée. Les règles de fonctionnement de ces programmes varient d'une prison à l'autre, mais les raisons évoquées restent les mêmes, à savoir d'éviter, autant que faire se peut, les conséquences néfastes d'un tel milieu sur les enfants (Association d'études et de recherches de l'École nationale de la magistrature, 1991; Bloom et Steinhart, 1993; Caddle et Crisp, 1997). En France, par exemple, une loi (Loi sur les prisons, 1977) prévoit que les prisons devront se doter des facilités nécessaires permettant aux mères d'accueillir leur(s) enfant(s) âgés de moins de 18 mois (Bertrand et al., 1998). En Allemagne, la cohabitation mère-enfant est permise jusqu'à l'âge de 
3 ans, et jusqu'à 6 ans dans les prisons «ouvertes», puisque les mères peuvent conduire leurs enfants à la garderie pendant la journée, et revenir avec eux à la prison le soir venu (Bertrand et al., 1998). Aux États-Unis, quelques prisons seulement ont un service de pouponnières permettant aux détenues de garder leur bébé jusqu'à l'âge de 18 mois.

Fait intéressant, en France et en Belgique, l'organisme communautaire Relais enfants-parents (REP) offre, depuis 1985, différents services permettant de maintenir ou de restaurer la relation parent-enfant malgré l'incarcération. En plus de faciliter la concertation entre les différents acteurs du système impliqués auprès des enfants et des parents détenus (homme et femme), le REP offre de la formation au personnel, des services de médiation et de counseling, le transport et l'accompagnement des enfants lors des visites, du soutien aux familles substituts, etc. (Relais enfants-parents, 1990). Dans certains États américains, il existe des centres correctionnels communautaires pour femmes pouvant accueillir des enfants d'âge préscolaire et servir d'alternatives à l'incarcération (Bloom et Steinhart, 1993; Bertrand et al., 1998).

À la lumière de ce qui précède, on observe que les pratiques et les conditions d'incarcération pour les femmes sont très diversifiées suivant les pays et les régions. Une telle disparité tient, entre autres, aux critères d'admissibilité de ces programmes, au nombre de places limitées ou encore à l'absence de responsabilité officielle reconnue au système pénal à l'égard des enfants des détenues (Macleod, 1986; Association d'études et de recherches de l'École nationale de la magistrature, 1991; Wine 1992; Bertrand et al., 1998).

Pourtant de nombreuses études tendent à démontrer qu'entre la moitié et les trois quarts des femmes incarcérées ont au moins un enfant (Macleod, 1986; Shaw, 1991; Wine, 1992; Bloom et Steinhart, 1993; Caddle et Crisp, 1997). Malgré l'absence de statistiques précises, MacLeod (1986) estime que, bon an mal an, environ 5000 enfants sont touchés par l'incarcération de leur mère au Canada. On sait également que près des deux tiers des femmes incarcérées sous juridiction fédérale ont déclaré avoir été, à un moment ou l'autre de leur vie, l'unique responsable de leur(s) enfant(s). Par conséquent, le seul fait d'être incarcérée peut s'avérer d'autant plus problématique et lourd de conséquences pour leur progéniture (SCC, 1990; Shaw, 1991; Watson, 1995).

En plus d'affecter un nombre important de femmes et d'enfants, cette question revêt de nombreuses dimensions. Au départ, l'incertitude et la confusion qui accompagnent une séparation génèrent de multiples pro- 
blèmes. Pour Macleod (1986), cela se révèle encore plus vrai pour une mère et son enfant en raison de l'importance du lien affectif qui les unit habituellement. Comme le souligne Labrecque (1995), la plupart des auteurs soulignent le fort attachement éprouvé par la plupart des enfants envers leurs parents biologiques, même en présence de problèmes familiaux graves, et le déchirement que représente pour eux une séparation. La séparation peut même engendrer chez certaines mères et certains enfants une détresse psychologique et physique (Macleod, 1986). L'on sait également à quel point la séparation et la perte des droits parentaux peuvent devenir une source de préoccupations importantes pour les mères, affectant du même coup leurs capacités d'adaptation en établissement et leurs chances de réhabilitation (Wine, 1992; Bloom et Steinhart, 1993).

A. Bouregba (1990), psychanalyste français, a bien fait ressortir les multiples enfermements et les difficultés personnelles que vivent les détenues qui sont mères. Si l'impression d'avoir abandonné leur enfant entraîne parfois le repentir ou le désir de réparer, elle peut aussi s'accompagner d'autodévalorisation, voire même de dépression. Plusieurs détenues éprouvent de la honte face à ce qui leur arrive, honte qui bien souvent se traduit par un sentiment de culpabilité et d'infériorité. Or, un parent honteux aura souvent peur de ne pas être à la hauteur ou de ne pas correspondre à l'image idéale qu'il s'est créée. Ne partageant plus le quotidien de leur enfant, ces femmes doivent arriver à faire le deuil de celui-ci. D'où, bien souvent, leurs hésitations et leurs conduites d'évitement face à leurs responsabilités parentales, maintes fois observées par les intervenants (Relais enfants-parents, $1990: 26$ ).

Par-delà le lien d'attachement qui existe entre une mère et son enfant, il n'en demeure pas moins que l'incarcération engendre de nombreuses difficultés : difficultés de contact en raison de l'éloignement, politiques carcérales restrictives au plan des visites et des communications, contraintes sécuritaires, absence de personnel spécialisé, soins inadéquats offerts aux femmes enceintes. De nombreuses études ont mis en évidence les lacunes et l'inadéquation des programmes par rapport aux multiples besoins des femmes (MacLeod, 1986; Shaw, 1991; Wine, 1992; Bloom et Steinhart, 1993).

En ce qui concerne les enfants, peu d'études ont porté exclusivement sur les conséquences de l'incarcération d'un parent. Un manque important se fait sentir au niveau des recherches longitudinales spécifiques aux enfants confrontés à la séparation de leur mère incarcérée et en ce qui concerne les facteurs de risques institutionnels (Richard, 1991; Laurence, 
1995 : 4). Le fait d'être séparé de sa mère peut, selon Gabel (1995), être une expérience traumatisante pour un enfant et mener à des problèmes de comportement chez une minorité non négligeable d'enfants. Bloom et Steinhart (1993) en ont identifié plusieurs : performance scolaire à la baisse, relations plus difficiles avec leurs pairs, difficultés générales de comportement, etc. Ces conséquences doivent toutefois être interprétées comme des facteurs dynamiques, multidirectionnels et non pas comme un processus linéaire obéissant à une relation de cause à effet. De nombreuses variables, telles les caractéristiques personnelles (âge, genre), familiales et la qualité du réseau familial élargi de l'enfant, peuvent interférer dans le processus de séparation. Il importe plutôt de chercher à comprendre les représentations que se forgent ces femmes et leurs enfants et voir comment la séparation peut affecter leur bien-être psychologique, matériel et social (MacLeod, 1986; Richards, 1991 : 4).

Pour neutraliser ces obstacles et éviter de briser le lien mère-enfant, certains proposent la cohabitation, alors que d'autres s'interrogent sur ces impacts. Cette question est ambiguë et ne fait pas nécessairement l'unanimité parmi les chercheurs (Lavergne, 1991). Pour certains, peu importe l'ajout de décorations, une prison demeure une prison et ne constitue pas un environnement adéquat pour élever un enfant, compte tenu de l'impact que pourrait avoir un tel climat sur son développement (Hunter, 1994). Dans la même veine, Labrecque (1995) souligne fort à propos à quel point le milieu environnant (c'est-à-dire le comportement des autres détenues) peut être stressant pour la mère et avoir un impact sur l'adaptation de l'enfant. Pour M. A. Bertrand et al. (1998), une telle pratique oblige des enfants à vivre en prison sans leur consentement et questionne le bien-fondé de cette forme d'élargissement du filet pénal.

\section{La démarche de recherche}

La plus grande partie de nos données de recherche provient d'un questionnaire soumis aux mères incarcérées dans les principaux établissements carcéraux et maisons de transition pour femmes au Québec. Les réponses obtenues, entre les mois de juillet et novembre 1999, nous ont permis de tracer le profil de ces femmes, de documenter la situation de garde de leurs enfants, de scruter les services auxquels elles ont recours, ainsi que l'appréciation qu'elles en font. Notre questionnaire comporte une soixantaine de questions et se divise en deux sections : la première porte sur les mères et la seconde sur leurs enfants, jusqu'à un maximum de trois. Toutes 
les mères étaient invitées à participer à cette recherche, sans égard à l'âge de leurs enfants et à la fréquence des contacts. Elles avaient également la possibilité de lire et de compléter le questionnaire dans l'une ou l'autre des deux langues officielles ou de nous dicter les réponses via un entretien. Le questionnaire comportait quelques questions ouvertes et des questions à choix multiples où elles avaient l'occasion également de développer et de préciser leurs réponses. Plusieurs de nos répondantes se sont prévalues de cette possibilité, nous offrant du même coup un matériel qualitatif fort pertinent que nous avons retranscrit intégralement.

Au total, nous avons obtenu la collaboration d'une centaine de femmes (voir tableau 1) et des réponses pour 203 de leurs enfants.

Au départ, il convient de signaler que la proportion de mères recensées parmi l'ensemble des détenues (70\%, soit 166 sur 237) s'avère conforme aux proportions qu'on retrouve dans les autres études sur ce sujet. Par ailleurs, avec un taux de participation s'élevant à tout près de $60 \%$, force est de conclure que notre approche s'est avérée efficace et que notre échantillon peut être considéré comme représentatif. La saisie des réponses inscrites aux questionnaires a été effectuée au fur et à mesure

TABLEA U 1

Distribution des femmes judiciarisées au Québec au sein des établissements visités

\begin{tabular}{|c|c|c|c|c|}
\hline & $\begin{array}{l}\text { Population totale } \\
\text { de femmes }^{\mathrm{a}}\end{array}$ & $\begin{array}{c}\text { Nombre de } \\
\text { mères }\end{array}$ & $\begin{array}{c}\text { Nombre de } \\
\text { répondantes }\end{array}$ & $\begin{array}{c}\text { Taux de } \\
\text { participation }\end{array}$ \\
\hline \multicolumn{5}{|l|}{ Milieu carcéral } \\
\hline $\begin{array}{l}\text { Établissement Joliette } \\
\text { (fédéral) }\end{array}$ & 65 & 46 & 38 & $82,6 \%$ \\
\hline $\begin{array}{l}\text { Maison Tanguay } \\
\text { (provincial) }\end{array}$ & 86 & 61 & 31 & $50,8 \%$ \\
\hline $\begin{array}{l}\text { Centre de Détention } \\
\text { féminin (provincial) }\end{array}$ & 45 & 33 & 18 & $54,5 \%$ \\
\hline \multicolumn{5}{|l|}{ Milieu communautaire } \\
\hline Maison Thérèse-Casgrain & 30 & 18 & 7 & $38,9 \%$ \\
\hline Expansion Femmes & 11 & 8 & 5 & $62,5 \%$ \\
\hline Grand total & 237 & 166 & 99 & $59,6 \%{ }^{c}$ \\
\hline
\end{tabular}

Notes:

a) Ces chiffres indiquent le nombre de femmes présentes au moment de la distribution du questionnaire.

b) Nombre approximatif (selon les informations disponibles).

c) Moyenne du taux de participation. 
que progressait notre tournée et nous avons eu recours au progiciel statistique SPSS pour le traitement des données quantifiables.

Lors de la distribution de notre questionnaire dans les différents établissements et maisons de transition, nous avons été témoin du fonctionnement de ces institutions et des activités auxquelles prennent part ces femmes. Afin de tirer le plus grand bénéfice de notre présence dans ces établissements, nous en profitions pour réaliser des entretiens informels avec le personnel de première ligne, lequel était bien placé pour nous informer du déroulement des activités, des services offerts, du nombre de mères sur les lieux, etc. D'autres échanges, via des entretiens semi-dirigés, ont été réalisés auprès de la direction d'une maison de transition, d'une travailleuse sociale de la Direction de la protection de la jeunesse (DPJ), d'un membre du contentieux de la DPJ, ainsi que d'une psychologue spécialisée en enfance. En dépit du faible nombre de professionnels ainsi rencontrés, cette démarche s'est révélée très utile et nous a permis de relativiser le point de vue des mères à l'étude et de développer une compréhension plus large des enjeux soulevés par cette problématique.

En vue de nuancer et d'intégrer l'information ainsi recueillie, nous avons également procédé à une démarche d'observation directe. La plus grande partie de notre travail d'observation s'est déroulée dans chacun des établissements où le questionnaire a été distribué, de même que dans différents endroits où les détenues pouvaient recevoir leur(s) enfant(s). À ces occasions, nous portions une attention particulière à tout ce qui relevait de la dynamique mère-enfant. Nous avons assisté, à titre d'observatrice, au programme sur les compétences parentales et familiales offert à l'établissement de Joliette. Consciente des éventuels effets que pouvait engendrer notre double statut (employée du SCC et chercheure), toutes les femmes ont été informées individuellement du caractère confidentiel et anonyme de cette recherche. Nous avons également obtenu au préalable leur autorisation écrite. Nous avons répété l'expérience d'observation à la Maison Tanguay, où nous avons assisté aux programmes de visites mère-enfant (visite au gymnase et à la roulotte) organisés par l'organisme communautaire Continuité famille auprès des détenues (CFAD).

Notre statut d'employée à l'établissement de Joliette, où nous occupions un poste d'intervenante de première ligne, a contribué sans aucun doute à l'avancement de nos connaissances. Nous avons travaillé, entre autres, au sein de l'unité de cohabitation mère-enfant et assisté, dans le cadre de nos fonctions, à la majorité des accouchements de mères prenant part à ce programme. Cependant, nous nous sommes interrogée sur 
l'impact de notre double statut sur les réponses des participantes et le traitement de nos données. Afin de minimiser les biais possibles, la cueillette des données s'est faite en dehors de nos heures de travail, les femmes étant avisées de notre présence sur les lieux en tant que chercheure et non en tant qu'employée. Nous avons insisté également sur l'aspect volontaire de leur participation, le caractère anonyme du questionnaire et la confidentialité entourant notre démarche de recherche. Le formulaire de consentement précisait que les informations recueillies ne pouvaient en aucun temps leur être préjudiciables ou nuire à la gestion de leur sentence. À chaque fois, les détenues ne semblaient y voir aucune objection et semblaient empressées de pouvoir partager leur vécu de mère. Nous avons également redoublé d'efforts pour maintenir une distance objective par rapport au traitement de nos données et des informations recueillies. Lors des séances d'observation, les réflexions suscitées étaient généralement notées la journée même dans un cahier de bord. Pour ces mêmes raisons, nous avons par ailleurs diversifié nos sources de données et les méthodes utilisées.

\section{Profil des mères incarcérées et de leur(s) enfant(s) au Québec}

Comme l'illustre le tableau 2, la majorité des femmes de notre échantillon ont entre 26 et 40 ans. À cet égard, nos données se trouvent à faire écho aux études canadiennes (Finn et al., 1999) et américaines (Bloom et Steinhart, 1993) qui situent l'âge moyen des femmes détenues au début de la trentaine.

TABLEAU 2

Répartition par groupe d'âge et par type de sentences des mères étudiées

\begin{tabular}{|l|c|c|c|c|}
\hline Groupe d'âge & $\begin{array}{c}\text { Sentence provinciale } \\
\text { (nombre) }\end{array}$ & Pourcentage & $\begin{array}{c}\text { Sentence fédérale } \\
\text { (nombre) }\end{array}$ & Pourcentage \\
\hline $18-21$ ans & 4 & $8,5 \%$ & 1 & $2,4 \%$ \\
\hline $22-25$ ans & 3 & $6,4 \%$ & 2 & $4,9 \%$ \\
\hline $26-30$ ans & 10 & $21,3 \%$ & 9 & $22,0 \%$ \\
\hline $31-40$ ans & 23 & $48,9 \%$ & 15 & $36,6 \%$ \\
\hline $41-50$ ans & 7 & $14,9 \%$ & 8 & $19,5 \%$ \\
\hline $51-60$ ans & - & - & 5 & $12,2 \%$ \\
\hline 61 ans et plus & - & - & 1 & $2,4 \%$ \\
\hline Total : & 47 & $100,0 \%$ & 41 & $100,0 \%$ \\
\hline
\end{tabular}


Lorsqu'on examine la distribution par groupe d'âge, on remarque que les seuls écarts relativement significatifs entre les femmes incarcérées dans des établissements provinciaux ou fédéraux ont trait aux mères qui se situent aux extrêmes de la pyramide des âges : les plus âgées se retrouvent plus facilement en pénitencier, alors que les plus jeunes sont nombreuses à être sous juridiction provinciale.

À l'instar de ce que d'autres études ont démontré (SCC, 1990; Biron, 1992; Wine, 1992), on constate que les femmes que nous avons rejointes comptent une forte proportion de chefs de famille monoparentale. Si toutes les femmes de notre échantillon ont des enfants, à peine $25 \%$ d'entre elles déclarent être mariées ou vivre en union de fait.

Les caractéristiques socioéconomiques des femmes de notre étude recoupent une fois de plus les données de diverses études : elles sont sous-scolarisées, n'ont guère une longue expérience de travail et déclarent de faibles revenus. À peine le tiers $(35,8 \%)$ des femmes de notre échantillon possèdent un diplôme de formation professionnelle, d'études secondaires ou post-secondaires.

Une minorité d'entre elles occupaient un emploi avant d'être incarcérées, si bien qu'un peu plus des deux tiers $(68,4 \%)$ étaient prestataires de revenus de la solidarité sociale (BES) préalablement à leur incarcération. Ces dernières touchaient alors une prestation moyenne de 633,89\$ par mois, soit l'équivalent d'un revenu annuel légèrement supérieur à $8300 \$$. Même en tenant compte de l'ensemble des revenus - ceux de l'éventuel conjoint, des prestations de la sécurité du revenu, des allocations familiales et des autres sources d'entrées de fond - près des deux tiers des mères à l'étude nous ont déclaré des revenus familiaux annuels inférieurs à 20000 \$. MacLeod (1986:22) faisait d'ailleurs valoir que les difficultés qu'éprouvent ces femmes à prendre soin de leur(s) enfant(s) ont d'abord pour source leur situation socioéconomique. Dans ce contexte, la perte de la garde de leur enfant et leur insertion dans des activités criminelles pourraient être vues comme des indicateurs d'instabilité sociale et financière grave.

Dans la mesure où ces caractéristiques peuvent avoir un impact sur leurs relations avec les enfants, nous avons tenté d'approfondir certaines questions d'ordre psychosocial. Nous nous retrouvons encore une fois en terrain familier et bien documenté. Un environnement social défavorisé, criminalisé et pauvre en ressources, un passé marqué d'abus physique et sexuel auquel viennent souvent se greffer des problèmes de toxicomanie (Bloom et Steinhart, 1993), voilà quelques-unes des caractéristiques qui 
se dégagent de nos données. Plus des trois quarts $(78,1 \%)$ des infractions déclarées par nos répondantes sont reliées — dans des proportions relativement similaires - aux infractions avec violence ${ }^{3}(28,5 \%)$, aux crimes reliés aux stupéfiants $(25,2 \%)$ et à ceux contre les biens $(24,4 \%)$. La prostitution ou la conduite avec facultés affaiblies comptent pour une faible proportion $(5,9 \%$ et $4 \%)$ des délits imputés à ces femmes.

Questionnées sur la possibilité d'un lien entre leurs activités criminelles et la consommation de substances intoxicantes, la majorité $(60,6 \%)$ des femmes répondent par l'affirmative. Environ les deux tiers de nos répondantes déclarent d'ailleurs avoir fait usage de substance intoxicante avant leur arrestation. L'alcool et la cocaïne sont les plus souvent cités. L'ensemble de nos données tend à montrer que plusieurs de ces femmes présentent des problèmes de toxicomanie, une situation pouvant notamment avoir une incidence sur le type de relations qu'elles entretiennent avec leur(s) enfant(s).

Sur un autre plan, près des deux tiers $(64,9 \%)$ des mères à l'étude ont déclaré avoir déjà été victimes d'abus sexuel ou d'inceste. Deux fois sur trois, l'agresseur aurait été un membre de la famille, bien souvent le père ou le beau-père. D'autres études ont d'ailleurs démontré qu'un part importante de toxicomanes avaient déjà vécu une situation d'abus (Bergeron, 1989).

Nos données montrent ensuite que $66,7 \%$ de nos répondantes ${ }^{4}$ avaient au moins un membre de leur famille immédiate et/ou élargie ayant des antécédents carcéraux. Le plus souvent, il s'agit de leur fratrie ou de la famille élargie. Il convient ici de noter que cette question peut avoir des incidences directes sur les mères incarcérées, notamment en matière de soutien. En effet, une personne qui présente des antécédents criminels peut se voir restreindre (visite guichet; demande refusée) l'accès aux visites en milieu carcéral. De plus, la DPJ peut lui refuser le statut de famille d'accueil (et la rétribution qui l'accompagne).

Lors de la réalisation de notre sondage, un peu plus de la moitié (52\%) des femmes de notre échantillon n'en étaient pas à leur première expérience du système judiciaire. Généralement, ces dernières avaient déjà été incarcérées pour une période inférieure à deux ans; celles au casier judiciaire le plus chargé pouvaient avoir derrière elles jusqu'à une vingtaine de

3. Les délits avec violence incluent les homicides, les tentatives de meurtre, les voies de fait et les vols qualifiés.

4. Il faut noter toutefois que nos données sur ce point sont très parcellaires et ne concernent que les réponses des détenues d'un seul établissement $(\mathrm{n}=31)$. 
sentences carcérales. De telles données soulèvent déjà de nombreuses questions quant aux impacts possibles des incarcérations à répétition sur la relation mère-enfant et sur le lien d'attachement que développent ces enfants avec d'autres personnes.

Si on observe la distribution d'ensemble des sentences imposées, celles-ci se répartissent dans des proportions relativement similaires entre les femmes purgeant une peine provinciale $(53,4 \%)$ et celles sous juridiction fédérale $(46,6 \%)$. La sentence la plus fréquemment imposée à l'ensemble des femmes de notre échantillon varie de six mois à un an, ce qui représente la situation d'une mère sur quatre et des séjours en temps réel purgé d'une durée maximum de 8 mois. En fait, près des deux tiers des sentences imposées varient entre 6 mois et 4 ans. Du côté des femmes purgeant une peine provinciale, on constate que les courtes sentences (de moins de 6 mois) représentent près de $30 \%$ de l'ensemble des peines de moins de deux ans. Quant à celles qui purgent une sentence fédérale, elles sont le plus souvent condamnées à une peine variant entre deux et trois ans. Suivent ensuite, en termes d'importance numérique, les femmes condamnées à une peine de trois à quatre années d'incarcération $(21,9 \%)$ et celles qui purgent une sentence à vie $(21,9 \%)$. À cet égard, un enfant dont la mère est condamnée à vie se retrouve nécessairement avec un deuil à faire : bien que cette dernière soit encore vivante, l'enfant sera séparé d'elle pour une période allant de dix à vingt-cinq ans. Étant donné la longueur de telles sentences, il est difficile de passer sous silence les besoins spécifiques en matière de maintien du lien et les conséquences d'une telle séparation pour ces mères et ces enfants.

Pour les 99 mères de notre échantillon, nous avons recensé un total de 203 enfants, soit une moyenne d'environ deux enfants par mère.

Comme l'illustre le tableau 3, les enfants âgés de 5 à 11 ans constituent la plus importante cohorte de notre échantillon, suivie par les enfants d'âge adulte. Néanmoins, lorsque l'on fait abstraction des enfants âgés de 18 ans et plus, on remarque que les deux tiers $(64,4 \%)$ des enfants sont d'âge scolaire (niveau primaire et secondaire). On parle ici d'enfants en âge de réaliser la situation d'incarcération de leur mère tant au niveau de la séparation engendrée que des enjeux entourant le maintien du lien. Moins nombreux, les enfants d'âge préscolaire représentent pourtant le groupe sur lequel se concentre la majorité des études concernant les conséquences d'une séparation avec la mère. De telles données sur l'âge des enfants nous permettent davantage de cibler les besoins au niveau des pratiques, surtout depuis que le SCC rend acces- 
TABLEA U 3

Âge des enfants des mères étudiées

\begin{tabular}{|l|c|c|c|}
\hline \multicolumn{1}{|c|}{ Catégorie d'âge } & Nombre & Pourcentage & $\begin{array}{c}\text { Distribution des enfants } \\
\text { de moins de 18 ans }\end{array}$ \\
\hline 0 à 4 ans & 46 & $23,3 \%$ & $32,6 \%$ \\
\hline 5 à 11 ans & 62 & $31,5 \%$ & $43,9 \%$ \\
\hline 12 à 17 ans & 33 & $16,7 \%$ & $23,5 \%$ \\
\hline 18 ans et + & 56 & $28,5 \%$ & - \\
\hline Sous-total & 197 & $100,0 \%$ & $100,0 \%$ \\
\hline Valeurs manquantes & 6 & & \\
\hline Grand total & 203 & & \\
\hline
\end{tabular}

sible le programme de cohabitation aux enfants jusqu'à l'âge de 13 ans, une première au niveau international.

En dépit de la situation carcérale, la majorité des enfants $(67,4 \%)$ demeurent sous la responsabilité légale de leur mère. Une précision s'impose toutefois : il ne faut pas confondre garde légale et vie commune, car il arrive souvent que l'enfant ait été placé bien avant l'incarcération de sa mère, à la suite d'une entente à l'amiable. D'ailleurs, près de la moitié des enfants $(49,2 \%)$ à l'étude ne vivaient pas avec leur mère au moment de l'incarcération de celle-ci. Cependant, le tiers d'entre eux était âgé de plus de 18 ans $^{5}$. On retrouve d'ailleurs ces mêmes données dans d'autres études (Macleod, 1986; Wine, 1992; Enos, 2001). Les enfants séparés de leur mère avant l'incarcération de celle-ci vivaient alors soit dans une famille d'accueil $(36,6 \%)$, soit chez leur père $(31,2 \%)$, soit chez un membre de la famille élargie $(30,2 \%)$.

Nous constatons ainsi que l'incarcération n'est pas toujours à l'origine de la rupture entre une mère et son enfant. En effet, les mères nous ont révélé que d'autres événements interviennent parfois en amont et justifient la prise en charge de leur(s) enfant(s) dans un milieu de vie substitut. Parmi les raisons évoquées par ces mères pour expliquer comment elles en sont venues à se séparer de leur(s) enfant(s), on retrouve : leurs problèmes de toxicomanie $(41,3 \%)$, l'intervention de la Direction

5. Sur un total de 195 enfants pour lesquels nous avons de l'information, 96 d'entre eux ne vivaient pas avec leur mère avant l'arrestation de celle-ci. Au total, 32 de ces enfants étaient âgés de plus de 18 ans. 
de la protection de la jeunesse (DPJ) (20,3\%), des difficultés récurrentes avec la justice $(16,5 \%)$ et d'autres motifs ${ }^{6}(21,5 \%)$.

\section{La relation mère-enfant en milieu carcéral}

Les enjeux entourant les relations mères-enfants durant l'incarcération sont nombreux et la question du maintien du lien comporte plusieurs incertitudes. Cela se comprend assez bien dans la mesure où ces relations évoluent au gré des circonstances qui surviennent dans la vie de la mère, des enfants ou des familles substituts. De plus, les interactions familiales sont souvent triangulées par les interventions des différents intervenants impliqués dans le dossier (travailleuse sociale de la DPJ, agent du milieu carcéral). L'examen proposé ici s'appuie donc sur les données de notre questionnaire, sur nos observations sur le terrain et sur les renseignements obtenus au cours d'entretiens réalisés auprès des différents acteurs concernés par la question.

Comme nous l'avons mentionné, il importe de ne pas associer trop rapidement les difficultés de ces femmes et de ces enfants au simple fait de l'incarcération. La situation des enfants placés en famille d'accueil par la DPJ en est un exemple. Plus du tiers $(36,6 \%)$ des enfants à l'étude se sont retrouvés dans cette situation avant même l'incarcération de leur mère, alors qu'à l'analyse de la situation d'ensemble, la proportion de ces enfants demeure relativement la même après l'arrestation de leur mère.

En raison des liens familiaux qui les unissent, l'alternative de choix pour ces mères demeure le placement de leur(s) enfant(s) chez des membres de la famille immédiate ou élargie. Tel que l'illustre le tableau 4, la majorité $(59,3 \%)$ des enfants se retrouve le plus souvent chez leur père, leurs grands-parents ou leurs oncles/tantes à la suite de l'arrestation de leur mère. Il est question bien souvent d'un placement temporaire, puisque la majorité $(64,3 \%)$ des mères à l'étude nous ont mentionné vouloir reprendre la garde de leur enfant dès leur libération.

L'analyse de l'appréciation des rapports entretenus par les mères avec les personnes assumant la garde de leur(s) enfant(s) permet de constater que les conflits ne sont pas rares et qu'ils surgissent fréquemment autour de l'organisation ou de la fréquence des contacts. Lorsque les personnes qui gardent les enfants s'opposent aux visites, plusieurs mères incarcérées

6. Dans la catégorie «autres», nous avons regroupé différentes situations : dépression de la mère, enfants vivant à l'étranger, enfant placé en vertu de la Loi sur les jeunes contrevenants, garde partagée... 
TAB LEA U 4

Les personnes assurant la garde des enfants après l'incarcération des mères étudiées

\begin{tabular}{|l|c|c|}
\hline \multicolumn{1}{|c|}{ Gardiens de l'enfant } & Nombre & Pourcentage \\
\hline Père & 37 & $23,9 \%$ \\
\hline Grands-parents & 34 & $21,9 \%$ \\
\hline Oncles/tantes & 21 & $13,5 \%$ \\
\hline Ami(es) & 7 & $4,5 \%$ \\
\hline Famille d'accueil via DPJ & 48 & $31,0 \%$ \\
\hline Autres & 8 & $5,2 \%$ \\
\hline Sous-total & 155 & $100,0 \%$ \\
\hline Ne s'applique pas & 39 & \\
\hline Valeurs manquantes & 9 & \\
\hline Grand total & 203 & \\
\hline
\end{tabular}

déplorent le manque de ressources pouvant les aider à maintenir des liens satisfaisants avec leurs enfants. Par exemple, si le père refuse les visites ou les appels téléphoniques, les recours de la mère s'avèrent fortement limités et nécessitent bien souvent l'intervention de la justice. De plus, en raison des liens d'attachement qui se développent entre l'enfant et sa famille de substitution, les conflits peuvent également survenir au sujet de la garde de l'enfant. Selon les antécédents (passé carcéral de la mère), l'âge de l'enfant ou la durée de la sentence, les personnes qui ont la garde de l'enfant peuvent demander que la situation soit clarifiée légalement. Si tel est le cas, ces dernières pourront, selon l'ordonnance de placement émise par la Cour du Québec (Chambre de la jeunesse), être reconnues comme famille d'accueil et ainsi rémunérées pour les soins apportés à l'enfant. En d'autres occasions, les conflits entre la mère et les gardiens de l'enfant sont absents, mais le maintien du lien est tout aussi fragile, compte tenu des moyens limités de ces derniers (état de santé précaire de la grand-mère, absence de moyens de transport, etc.).

On le devine facilement, de telles situations ouvrent la porte à des relations conflictuelles où l'enfant se retrouve au centre des litiges. Bref, les enjeux sont multiples et ne sont pas sans conséquence pour la relation mère-enfant. Nous verrons maintenant comment le système carcéral répond à l'heure actuelle aux besoins de ces mères et de ces enfants.

Parmi les services institutionnels les plus utilisés par les femmes de notre échantillon pour maintenir le lien avec leur(s) enfant(s), on remarque 
que les échanges téléphoniques $(42,6 \%)$ et épistolaires $(25,4 \%)$ sont le plus souvent cités. Quant aux programmes de visites en milieu carcéral (tous types confondus), il est surprenant de constater qu'ils représentent seulement le quart $(24,6 \%)$ de l'ensemble des services utilisés par ces mères. De plus, on constate que près de $15 \%$ des enfants n'entretiennent aucun contact avec leur mère ${ }^{7}$ durant son incarcération.

En fait, plusieurs mères s'interrogent sur le bien-fondé des règlements entourant les programmes de visites régulières. Nous avons remarqué que ces règlements different largement d'un établissement à un autre et ont un impact sur la qualité et la fréquence des contacts mère-enfant. Par exemple, les établissements provinciaux visités imposent que les premières visites pour les détenues nouvellement admises soient sécuritaires (derrière une vitre, sans possibilité de contacts physiques), une pratique qui expose les enfants à des conditions fort déplorables, susceptibles même de laisser des séquelles et d'influer sur la fréquence de leurs visites. Pour éviter de telles conditions, les mères et leur(s) enfant(s) devront composer avec des délais d'attente supplémentaires avant de bénéficier de visites contacts, soit la complétion du nombre minimal de visites sécuritaires par l'adulte qui accompagnera ultérieurement l'enfant. Qui plus est, le nombre de visites accordé par mois (6 à 8 visites au provincial et nombre illimité au fédéral), le temps accordé par visite ( 2 à 8 heures) et les heures d'ouverture du département des visites sont d'autres exemples qui illustrent la disparité des pratiques correctionnelles selon le lieu d'incarcération et exposent dès lors ces femmes et enfants à des conditions de visites fort variables. Force est de constater que les installations qu'on retrouve au sein des départements des visites carcérales tiennent rarement compte des besoins des enfants. L'établissement de Joliette possède toutefois, à même son parloir des visites, une salle de jeux pour les enfants et une cour extérieure.

Pour pallier ces contraintes organisationnelles et répondre aux besoins familiaux des détenues, deux des trois établissements visités offrent aux femmes la possibilité de recevoir la visite de leur(s) enfant(s) dans un environnement adapté. À l'établissement de Joliette, grâce à la présence d'une psychologue ${ }^{8}$ qui coordonne le programme de maintien du lien

7. Il est à noter que les répondantes étaient invitées à nous expliquer les différentes formes de liens qu'elles entretenaient avec chacun de leurs enfants. Au total, nous avons recensé 272 mentions.

8. Des cinq établissements régionaux, seul l'établissement de Joliette offre les services à temps plein d'une psychologue spécialisée en enfance. 
mère-enfant, plusieurs activités existent pour répondre non seulement aux besoins des mères et de leurs enfants, mais également à ceux des familles de substitution ${ }^{9}$. Les personnes significatives dans la vie de l'enfant sont invitées à prendre part à ces visites qui s'actualisent au gymnase du pénitencier et à la garderie munie d'une cuisine et d'une cour extérieure. On retrouve entre autres comme activités les visites-gymnases mensuelles, les activités thématiques selon les saisons ou les festivités (anniversaire de l'enfant, fête des mères) et le programme sur les compétences parentales et familiales. De tels services s'avèrent possibles grâce à la présence à temps plein d'une psychologue, seul effectif spécialisé en enfance parmi les établissements pour femmes visités. À la prison Tanguay, les visites mères-enfants s'actualisent par l'entremise des intervenants de l'organisme communautaire Continuité famille auprès des détenues (CFAD). Elles se déroulent en avant-midi, tous les dimanches. Les enfants, jusqu'à l'âge de16 ans, peuvent ainsi se livrer à différentes activités au gymnase : dessin, ping-pong, jeu de Mississipi, volley-ball et autres. Le transport des enfants est assuré par les intervenants du CFAD et facilite les contacts. De telles visites favorisent la qualité des relations mèresenfants et sont grandement appréciées par toutes les parties. Toutefois, les départs font bien souvent l'objet de réactions émotives et justifient la présence d'intervenants spécialisés pour encadrer ces visites et apporter l'aide et le soutien demandés par certains enfants et mères qui, en larmes, s'inquiètent et demandent déjà quand aura lieu la prochaine visite.

Quant aux programmes permettant aux enfants de séjourner quelques jours avec leur mère dans des unités à l'écart du reste de la population carcérale, on les retrouve à la Maison Tanguay (Montréal) et à l'établissement de Joliette. Ils représentent $6,6 \%$ de tous les contacts mentionnés par les mères pour maintenir le lien avec leur(s) enfant(s). Le premier s'actualise par l'entremise de l'organisme CFAD et permet aux enfants âgés de moins de 16 ans de passer la nuit (visite de 24h) avec leur mère dans une roulotte. Deux mères détenues y séjournent à la fois avec leur(s) enfant(s). La roulotte qui les accueille est ceinturée d'une clôture et d'espaces de jeux extérieurs. Elle date toutefois des années $1970^{10}$, et des rénovations sont à prévoir pour assurer la continuité d'un tel service. Les

9. Si autorisé sur la liste des visiteurs de la mère, à l'exception du personnel de la DPJ assistant parfois aux visites.

10. Cette roulotte servait, jusqu'en 1997, aux détenues sous juridiction fédérale qui purgeaient leur peine à la Maison Tanguay (selon l'entente fédérale-provinciale), ainsi qu'à leur famille. Le programme de roulotte CFAD avait lieu en semaine. 
intervenants du CFAD assurent en totalité la coordination du programme; ils assument, durant ces visites, non seulement la liaison avec les intervenants de la DPJ et les familles de substitution, mais également avec le personnel correctionnel de la Maison Tanguay. À l'établissement Joliette, le programme des visites familiales privées (VFP) s'actualise de façon bien différente : absence de personnel sur les lieux ${ }^{11}$, séjours plus longs (72 heures), possibilité de recevoir jusqu'à cinq membres de leur famille ou de se prévaloir de moments plus intimes, seules avec leur(s) enfant(s) ${ }^{12}$. Les deux unités de VFP accessibles sont de construction récente et spacieuses. Cependant, les cours extérieures sont très exiguës et ne permettent pas aux enfants qui y séjournent de s'amuser librement. C'est d'ailleurs une des principales critiques faites par les mères étudiées.

En plus de ces différents services, le programme de cohabitation mèreenfant est accessible, depuis le mois d'août 1998, aux femmes purgeant une peine fédérale. Au moment de notre étude, dix femmes nous ont mentionné leur expérience de grossesse. Quatre d'entre elles ont pu profiter de ce programme et éviter la séparation d'avec leur nouveau-né. Les six autres femmes, incarcérées sous juridiction provinciale, ont dû terminer leur sentence carcérale et être séparées de leur enfant quelques jours après lui avoir donné naissance, ce qui n'est pas sans conséquence dans la vie d'une femme. La moitié de ces enfants ont dû être placés par la DPJ dans une famille d'accueil et les autres, chez leurs grands-parents. Notons que le quart des mères à l'étude ont déclaré avoir déjà vécu l'expérience d'une grossesse en milieu carcéral; parmi celles-ci, moins de la moitié ont accouché avant d'être libérées.

En fait, entre le mois de mai 1998 et le mois de décembre $1991^{13}$, quatre mères ont bénéficié du programme de cohabitation mère-nouveauné à l'établissement de Joliette, pour des séjours variant entre 5 semaines et près de 20 mois. En principe, une telle cohabitation est possible jusqu'à ce que l'enfant ait atteint l'âge de 4 ans. Quant à la cohabitation à temps partiel, 11 enfants âgés de 3 à 12 ans ont pu cohabiter avec leur mère $(\mathrm{n}=6)$ pour des séjours moyens de 3 jours, à une fréquence variable. Ces enfants séjournent au sein de l'unité mère-enfant et peuvent circuler, en compagnie de leur mère seulement, sur le vaste terrain du pénitencier.

11. Hormis les rondes faites lors des dénombrements officiels quatre fois par jour.

12. Les mères peuvent séjourner seules avec leur enfant si l'évaluation de la psychologue au programme de maintien du lien mère-enfant ne détermine aucun risque pour la sécurité des enfants.

13. Statistiques fédérales non publiées et recensées par M.-A. Nantel (2000). 
Rares sont les occasions où plusieurs enfants participent en même temps à ce programme de cohabitation. D'ailleurs, depuis l'ouverture de l'unité mère-enfant, cette dernière a dû fermer ses portes pour quelques mois, faute de participants. Une réalité qui n'est pas sans conséquence sur l'offre de soins et de services.

Au delà des services et programmes impliquant la présence des enfants en prison, il existe des programmes de permissions de sortie ou d'absences temporaires (avec ou sans surveillance) permettant aux femmes détenues de quitter temporairement la prison. Bien que ces programmes soient offerts dans tous les établissements visités, rares sont les femmes qui en bénéficient pour maintenir le lien avec leur enfant. En effet, le programme ne représente que $4,4 \%$ des services les plus utilisés. Pourtant la Loi sur le système correctionnel stipule que tout détenu (sous juridiction provinciale comme fédérale) peut se prévaloir de telles permissions de sortie pour des périodes pouvant aller jusqu'à 5 jours consécutifs. D'ailleurs, sous juridiction fédérale, un des motifs d'octroi reliés à ces permissions de sortie est d'encourager les délinquants à entretenir des rapports avec leur famille ou d'assumer leurs responsabilités parentales. Ces programmes de sortie pourraient être utilisés davantage pour permettre aux femmes de maintenir ou favoriser le lien avec leur progéniture plutôt que de se limiter aux visites des enfants en milieu carcéral.

En ce qui à trait à la surveillance des femmes en communauté, certaines d'entre elles doivent séjourner dans l'une des deux maisons de transition au Québec, soit la maison Thérèse-Casgrain et Expansion Femmes. Ces dernières accueillent à la fois les mères sous juridiction fédérale et provinciale. La cohabitation mère-enfant y est possible pour les enfants d'âge préscolaire. Peu de services spécifiques à la réalité de ces mères et enfants se sont toutefois développés. L'absence de budget pour couvrir les frais de garde des enfants est un autre motif évoqué par le personnel interrogé en maison de transition pour justifier cet état de fait. Une autre des contraintes est le petit nombre de femmes qu'on y retrouve, ce qui n'est pas sans conséquence sur la prestation de programmes. Le CRC Expansion femme dispense un programme sur les compétences parentales lorsque le nombre est suffisant. La seule ressource communautaire spécialisée pour les mères est l'organisme CFAD, ayant pignon sur rue à Montréal. Les services sont offerts durant la semaine et visent à faciliter les démarches d'intégration sociale des mères libérées ou ex-détenues. Des camps de jours sont également organisés pour les enfants. La survie de cette ressource est cependant liée aux subventions et aux dons reçus annuellement. 
Comme plus de la moitié $(55,8 \%)$ des mères de notre échantillon n'ont recours à aucun service d'aide et de soutien en lien avec leur situation de mères détenues ou libérées conditionnellement, nous avons invité cellesci à préciser quels étaient les principaux obstables pouvant limiter les contacts avec leurs enfants. Les deux tiers de leurs réponses $(66,3 \%)$ ont trait à des problèmes d'ordre matériel et financier : frais d'interurbains, possibilité de ne faire des appels téléphoniques qu'à «frais virés ${ }^{14}$, frais de déplacements des enfants, éloignement, absence de moyen de transport et règlements de l'établissement. Pour d'autres mères, les obstacles découlent des restrictions imposées par la Direction de la protection de la jeunesse et des résistances de la famille de substitution $(26,7 \%$ des réponses). Plus de la moitié des mères $(56,3 \%)$ qualifient leur relation avec la DPJ d'insatisfaisantes. Elles se sentent souvent menacées par le pouvoir décisionnel octroyé aux travailleurs sociaux (l'ordonnance de placement d'un juge de la Cour du Québec ordonne habituellement que les visites parents/enfants soient fixées par le Directeur de la protection de la jeunesse) et craignent qu'on ne leur retire la garde de leur enfant. D'autres obstacles, moins nombreux ( $7 \%$ des mentions), sont reliés à des raisons personnelles ou à d'autres motifs comme, par exemple, le désir de cacher à leur enfant leur statut de détenue ou encore le refus que leur enfant soit confronté à un tel milieu.

\section{Conclusion}

Bien que l'incarcération ne soit pas le seul facteur explicatif à la séparation mère-enfant, elle ne fait qu'accentuer une problématique déjà non négligeable. Nous l'avons vu, le profil des mères incarcérées au Québec démontre bien les difficultés auxquelles plus de la moitié d'entre elles doivent faire face : situation monoparentale, problèmes de consommation de substances intoxicantes, ressources financières limitées, expérience antérieure de séparation avec leur(s) enfant(s), etc. Quoi qu'il en soit, pour ces femmes, une réinsertion sociale réussie et la qualité de vie de leurs enfants passent presque toujours par des relations familiales satisfaisantes. On constate toutefois que les services et programmes actuels demeurent des initiatives isolées et sujettes à de multiples remaniements, étant donné

14. Notons que l'ensemble des établissements visités permettent aux mères d'effectuer un appel (ligne directe) à leur(s) enfant(s) à une fréquence bimensuelle et que certains établissements (Centre de détention féminin (CDF) et Maison Tanguay) assument les frais d'interurbain. 
l'absence de stratégies correctionnelles d'actions concertées et de politiques chargées de veiller aux besoins familiaux des personnes incarcérées pour le meilleur intérêt des enfants.

La question du maintien du lien mère-enfant en contexte carcéral est d'autant plus complexe à cerner que se rencontrent des intérêts divergents et de nombreux acteurs. À une époque où l'accent est mis sur le développement des programmes novateurs et sur des mesures de réinsertion sociale, il est surprenant de constater qu'on se bute toujours à des obstacles d'ordre organisationnel et financier, lesquels varient considérablement selon les établissements de détention! Les programmes novateurs de cohabitation ont certes leur place, mais il faut se rappeler que les conséquences à long terme sur la dyade mère-enfant sont encore imprécises et que des études sur les facteurs de risque en milieu carcéral sont à développer. Compte tenu des besoins de la clientèle et des enjeux présents, il serait souhaitable d'investir auprès d'un personnel spécialisé et de créer un partenariat entre les divers organismes sociaux (service correctionnel provincial et fédéral, DPJ, organismes communautaires et ressources actuelles), afin d'assurer la continuité des interventions et le développement d'une expertise pluridisciplinaire sur la question.

\section{Références}

Association d'études et de recherches de l'École nationale de la magistrature (1991). La mère détenue et son enfant. Bordeaux : Éditions Bergeret.

Bergeron, É. (1989). Toxicomanie chez les femmes. Bulletin d'information : InfoToxico-04. Centre d'accueil Domrémy Trois-Rivières, 1 (2).

Bertrand, M.-A., Biron, L., di Pisa, C., Fagnan, A.B., \& McLean, J. (1998). Prisons pour femmes. Montréal : Éditions du Méridien.

Biron, L. (1992). Les femmes et l'incarcération, le temps n'arrange rien. Criminologie, 25 (1), 119-130.

Bloom, B., \& Steinhart, D. (1993). Why punish the children? A reappraisal of the children of incarcerated mothers in America. États-Unis : National Council on Crime and Delinquency.

Caddle, D., \& Crisp, D. (1997). Imprisoned women and mothers. London : Home Office Research and Statistics Directorate.

Finn, A., Trevethan, S., Carrière, G., \& Kowalski, M. (1999). Les femmes détenues, détenus autochtones et détenus condamnés à perpétuité : un profil instantané d'une journée. Juristat, 19 (5). Centre canadien de la statistique juridique, Statistique Canada : Ottawa.

Gabel, S. (1995). Les problèmes de comportement des enfants dont les parents sont incarcérés. Forum, 7 (2), 37-39. 
Hunter, S.M. (1984). The relationship between women offenders and their children. Michigan State University, Thèse de doctorat.

Labrecque, R. (1995). Étude sur le programme mère-enfant. Rapport présenté au Service correctionnel du Canada.

Lavergne, C. (1991). La séparation mère enfant à la suite d'une incarcération : une mesure qui touche au moins $50 \%$ des femmes incarcérées au Canada. Femmes et Justice, 6 (2).

Maclean, D. (1997). Mother and child live-in programs in canadian prisons: A policy analysis of program development and implementation. Simon Fraser University, M.A. Paper.

Macleod, L. (1986). Condamnés à la séparation: Une étude des besoins et des problèmes des délinquantes et de leurs enfants. Ottawa : Division de la recherche, Direction des programmes, ministère du Solliciteur général du Canada.

Nantel, M.A. (2000). Compilation maison du programme mère enfant à l'Établissement Joliette. Service correctionnel du Canada : document inédit.

Relais Enfants-Parents (1990). Relais Enfants-Parents : entre l'enfant et ses parents, la prison. Montrouge, France : Colloque Maison de la Culture de Bourges (22 mars 1990).

Richards, M. (1991). The separation of children and parents: some issues and problems. In R. Shaw (ed.), Prisoner's children. What are the issues (pp.312). Library of Congress Cataloging in Publication Data.

Service correctionnel du Canada (1990). La Création de Choix. Groupe d'étude sur les femmes purgeant une peine fédérale. Ottawa : Ministère du solliciteur général du Canada.

Shaw, M. (1991). Sondage auprès des femmes purgeant une peine de plus de deux ans. Rapport pour Spécialistes : Ministère du Solliciteur général du Canada.

Watson, L. (1995). Dans l'intérêt de l'enfant : le programme mère-enfant. Forum, $7(2), 25-27$.

Wine, S. (1992). A motherbood issue: The impact of criminal justice system involvement on women and their children. Corrections Branch, Ministry of the Solicitor General of Canada. 